\title{
The relativistic shift of narrow spectral features from black-hole accretion discs
}

\author{
T. Pecháček ${ }^{1}$, M. Dovčiak ${ }^{2}$, V. Karas ${ }^{1,2}$, and G. Matt ${ }^{3}$ \\ ${ }^{1}$ Charles University, Faculty of Mathematics and Physics, V Holešovičkách 2, 18000 Prague, Czech Republic \\ 2 Astronomical Institute, Academy of Sciences, Boční II, 14131 Prague, Czech Republic \\ e-mail: vladimir.karas@cuni.cz \\ 3 Dipartimento di Fisica, Università degli Studi “Roma Tre”, via della Vasca Navale 84, 00146 Roma, Italy
}

Received 17 May 2005 / Accepted 14 June 2005

\section{ABSTRACT}

Transient spectral features have been discovered in the X-ray spectra of Active Galactic Nuclei, mostly in the 5-7 keV energy range. Several interpretations were proposed for the origin of these features. We examined a model of Doppler boosted blue horns of the iron line originating from a spot in a black hole accretion disc, taking into account different approximations of general relativistic light rays and the resulting shift of energy of photons. We provide a practical formula for the blue horn energy of an intrinsically narrow line and assess its accuracy by comparing the approximation against an exact value, predicted under the assumption of a planar accretion disc. The most accurate approximation provides excellent agreement with the spot orbital radius down to the marginally stable orbit of a non-rotating black hole.

Key words. line: profiles - accretion, accretion discs - black hole physics - X-rays: galaxies

\section{Introduction}

$\mathrm{X}$-rays are the main source of information about the innermost parts of accretion discs in active galactic nuclei (AGN) and Galactic black hole systems. The iron $\mathrm{K} \alpha$ line is the most prominent spectral feature, occurring at a rest energy $E_{0}$ ranging from $\sim 6.4 \mathrm{keV}$ (neutral iron) to $\sim 6.96 \mathrm{keV}$ (H-like iron). The natural width of these lines is only a few eV (Thompson et al. 2001). They are thought to originate as a result of illumination of an accretion disc, which is formed by relatively cold material, by high-energy continuum photons from a corona or a jet above the disc (Fabian et al. 2000; Reynolds \& Nowak 2003). The $\mathrm{K} \alpha$ lines are multiplets. The neutral iron line is a doublet with energies $E_{\mathrm{K} \alpha_{1}}=6.404 \mathrm{keV}, E_{\mathrm{K} \alpha_{2}}=6.391 \mathrm{keV}$. The small energy difference makes the doublet unresolved with present spectrometers, though it may be resolvable by ASTRO-E2 in the near future. Another prominent spectral feature, namely the $\mathrm{K} \beta$ line, arises at an energy of $\sim 7.07 \mathrm{keV}$ and the iron edge occurs at $7.1 \mathrm{keV}$. Special and general relativity effects strongly modify the appearance of all these features. For instance, the continuum becomes broader with increasing disc inclination due to enhanced Doppler shifts. The photoelectric edge is blurred into broad troughs. X-ray spectroscopy can then be employed to reveal the effects of general relativity influencing the observed energy and thereby shaping the spectral lines and the continuum slope.

Fast circulation of gas is the dominant source of line broadening and forms, together with substantial gravitational redshift, the observed profile. A double-horn shape is expected in the simplest case of a geometrically thin, Keplerian disc (Fabian et al. 1989), and the discovery of this kind of profile has been indeed reported in several objects, the best-known examples being the Seyfert galaxy MCG-6-30-15 (Tanaka et al. 1995) and the microquasar GRS 1915+105 (Martocchia et al. 2002), where the line shows equivalent widths reaching $\sim 200 \mathrm{eV}$. It has been argued that fine substructures of this broad feature could be used to constrain the inclination angle of the source, radial emissivity in the disc plane, and even the angular momentum of the central black hole (see Martocchia et al. 2000; Beckwith \& Done 2004, and references cited therein). However, very broad "classical" double-horn relativistic lines are often absent in AGN (e.g. Bianchi et al. 2004), and it is important to seek additional evidence.

In recent years, narrow emission features have been reported in the X-ray spectra of several AGNs: see Turner et al. (2002, 2004, 2005); Guainazzi (2003); Yaqoob et al. (2003); Bianchi et al. (2004); Dovčiak et al. (2004a); Gallo et al. (2004); McKernan \& Yaqoob (2004); Porquet et al. (2004); Della Ceca et al. (2005). This topical discovery renewed the interest in accretion disc hot spots. The narrow lines we have on mind are typically found in the 5-7 keV energy range, i.e. mostly on the redshifted side of the iron line rest energy. A tentative explanation for these puzzling features, which we adopt in this paper, is in terms of iron emission, which originates in a localized area of the disc $\left(\delta r \lesssim r_{\mathrm{g}}\right)$. According to this scheme the spectral features are produced by transient magnetic flares 
briefly illuminating the disc and producing the iron line by fluorescence in the surface layer (Haardt et al. 1994; Poutanen \& Fabian 1999; Czerny et al. 2004).

The idea of revealing the signatures of black holes via spectroscopy of orbiting spots has great potential and a long history (Cunningham \& Bardeen 1973). Theoretical line profiles have been studied including specific effects of general relativity: the frame-dragging (Kojima 1991; Laor 1991; Bromley et al. 1997), extreme light-bending and multiple images (Matt et al. 1993; Viergutz 1993; Bao et al. 1994; Zakharov \& Repin 2003; Beckwith \& Done 2005), non-axisymmetric (spiral) waves in accretion discs (Karas et al. 2001; Hartnoll \& Blackman 2002), and self-gravity (Karas et al. 1995; Usui et al. 1998). Various applications have been proposed for the general-relativity energy shift of spectral features from co-rotating spots; in particular, it was suggested that the range of energy fluctuations could constrain the source geometry and discrimate between planar and thick discs (Bao \& Stuchlík 1993).

In this paper we examine extremal values (maximum and minimum) of observed energy of a line from an orbiting spot. The main purpose of this study is to provide a simple, practical and accurate formula that will be useful in analyzing the redshifted narrow lines. We neglect light bending, because its influence is important for only a relatively minor fraction of photons on special rays, i.e. those passing near caustics. This approximation was assessed by Gerbal \& Pelat (1991), but these authors did not aim to provide an explicit formula that would be convenient for practical purposes. Our approach is similar to Zhang \& Bao (1991), who also ignored light bending. We improve their treatment of light aberration and this allows us to express the redshift function (defined as a ratio of observed to emitted energy; see Eq. (8) below) in a way that is comparably simple to but more accurate than their Eq. (2.9). As a further step, we include light bending in terms of Beloborodov's (2002) approximation and reach fractional accuracies of a few percent or better for the redshift function; the extremal values are given with relative error better than $0.1 \%$. Thus the resulting shift of energy is estimated at least an order of magnitude more precisely than before, and the error of its extremal values is negligible in most practical cases.

In the next section we summarize the main results of this paper. In particular, in Sect. 2.1 we specify our assumptions and give a prescription for calculating the observed energy range spanned by the spectral line (i.e. extremal values of $g$-factor). It will be of interest to a reader seeking a straightforward approach to practical problems of data fitting. Then, in Sect. 2.2, we describe the individual steps of the derivation, including a comparison with previous results of other authors. In Sect. 3, in a brief re-discussion of several AGNs we illustrate the interpretation of narrow spectral features in terms of the blue horn produced by an orbiting spot. The results presented here are limited to static black holes and geometrically thin, Keplerian discs. Even with these limitations, they may be useful in interpreting the present data and deriving disc parameters. As it will be shown in Sect. 3, the narrow features detected so far do not require spinning black holes.

\section{Calculation of the redshift}

\subsection{Summary}

For the gravitational field of the system we assume a nonrotating black hole and so adopt the Schwarzschild spacetime metric (Misner et al. 1972),

$\mathrm{d} s^{2}=-\frac{r-2}{r} \mathrm{~d} t^{2}+\frac{r}{r-2} \mathrm{~d} r^{2}+r^{2}\left(\mathrm{~d} \theta^{2}+\sin ^{2} \theta \mathrm{d} \phi^{2}\right)$,

where the radius $r$ is evaluated in units of gravitational radius $r_{\mathrm{g}} \equiv G M c^{-2} \doteq 1.5 \times 10^{5}\left(M / M_{\odot}\right) \mathrm{cm}$, with $M$ being the mass of the black hole. In Eq. (1) as well as everywhere in this text, geometrized units with $c=G=1$ are used; distances are thus made dimensionless by scaling them with $M$. Notice that the accretion disc own mass can be neglected, i.e. $M_{\mathrm{d}} \ll M$, because we constrain ourselves to the innermost regions of the flow where the disc is non-self-gravitating (in terms of Toomre's criterion).

The disc is assumed to be Keplerian and extending down to the marginally stable orbit, $r_{\mathrm{ms}}=6$, below which it ceases to exist. An observer is located at infinity on the $x$ axis (i.e. $\phi=0^{\circ}$ ). By approximating trajectories of light according to Beloborodov (2002) and evaluating the effect of aberration as detailed in Sect. 2.2, we obtain the extremal values of the energy shift in the form

$g_{\mathrm{B}}^{ \pm}=\frac{r^{1 / 2}(r-3)^{1 / 2}}{r+\left[r-2+4\left(1+\cos \phi_{ \pm} \sin \theta_{\mathrm{o}}\right)^{-1}\right]^{1 / 2} \sin \phi_{ \pm} \sin \theta_{\mathrm{o}}}$

These extremal values occur for the azimuthal polar coordinate $\phi \equiv \phi_{ \pm}$, defined by

$\sin \phi_{ \pm}=\mp\left(1-\cos ^{2} \phi_{ \pm}\right)^{1 / 2}$,

$\cos \phi_{ \pm}=f_{1}^{-1}\left[f_{2}{ }^{1 / 3}+(r-4)^{2} f_{2}{ }^{-1 / 3}-2(r-1)\right]$

$\left(\phi=90^{\circ}\right.$ corresponds to the maximally receding part of the disc $)$. The functions $f_{1}\left(r, \theta_{\mathrm{o}}\right)$ and $f_{2}\left(r, \theta_{\mathrm{o}}\right)$ are

$f_{1}\left(r, \theta_{0}\right)=3(r-2) \sin \theta_{0}$,

$f_{2}\left(r, \theta_{0}\right)=(r-4)^{3}+f_{3}^{2}+\left[2(r-4)^{3}+f_{3}{ }^{2}\right]^{1 / 2} f_{3}$,

with $f_{3}\left(r, \theta_{\mathrm{o}}\right)$ being

$f_{3}\left(r, \theta_{0}\right)=3 \sqrt{3}(r-2) \cos \theta_{0}$.

Equation (2) can be used to determine the range of energy spanned by a line without the need to evaluate special functions (elliptic integrals and their inverse in case of Schwarzschild black hole; e.g. Chandrasekhar 1992; Čadež et al. 2003) or to perform numerical ray-tracing in the curved spacetime. Instead, purely algebraic formulae are involved, allowing us to give extremal values of redshift as functions of the spot radius $r$ and observer's inclination angle $\theta_{\mathrm{o}}$. Below we estimate the accuracy of our approximation and find it very precise even at large values of inclination (almost edge-on, i.e. $\theta_{\mathrm{o}} \sim 89^{\circ}$ ) and small radii (down to the marginally stable orbit, $r \sim r_{\mathrm{ms}}$ ). We find much better agreement between approximate and exact values than what can be achieved with the Zhang \& Bao (1991) approach. 


\subsection{Details of the calculation}

In this section we derive the results and provide comparison with previous works. The relativistic shift $g$ of the energy of photons, i.e. the ratio of the frequency observed by a distant observer to the frequency emitted in a local frame co-rotating with the disc material, can be expressed, within the approximation of geometrical optics, in terms of the four-momentum of photons $p$ and the four-velocity $U$ of the emitting material and of the distant observer. The shift $g\left(r, \phi ; \theta_{\mathrm{o}}\right)$ is a function of polar coordinates in the disc plane and of observer's inclination,

$g\left(r, \phi ; \theta_{\mathrm{o}}\right) \equiv \frac{E_{\mathrm{o}}}{E_{\mathrm{e}}}=\frac{p_{\mathrm{o} \mu} U_{\mathrm{o}}^{\mu}}{p_{\mathrm{e} \alpha} U_{\mathrm{e}}^{\alpha}}=-\frac{p_{\mathrm{o} t}}{p_{\mathrm{e} \alpha} U_{\mathrm{e}}^{\alpha}}$,

where $E_{\mathrm{o}}$ denotes the photon energy measured by a distant static observer $(r \rightarrow \infty), E_{\mathrm{e}}$ is the locally emitted energy from a source co-rotating with the disc, $U_{\mathrm{e}}^{\mu}$ is the four-velocity of the disc medium, and $U_{\mathrm{o}}^{\alpha}$ is the observer's four-velocity.

Equation (8) can be written in the following way:

$g\left(r, \phi ; \theta_{\mathrm{o}}\right)=\frac{1}{U_{\mathrm{e}}^{t}(r)\left[1-\Omega(r) l\left(r, \phi ; \theta_{\mathrm{o}}\right)\right]}$,

where $U_{\mathrm{e}}^{t}(r)=(1-3 / r)^{-1 / 2}$ is a component of the disc Keplerian four-velocity, $\Omega(r)=r^{-3 / 2}$ is the angular velocity and $l\left(r, \phi ; \theta_{0}\right)$ is the azimuthal component of the photon's angular momentum (constant along null geodesics). The dependence $l\left(r, \phi ; \theta_{0}\right)$ on the polar coordinates in the disc can be calculated analytically with the help of elliptic functions (Chandrasekhar 1992). Let us adopt the approximation in terms of the $\phi$-component of the photon momentum in a local frame,

$l\left(r, \phi ; \theta_{\mathrm{o}}\right)=\frac{n^{(\phi)}\left(r, \phi ; \theta_{\mathrm{o}}\right) e_{(t)}^{t}(r)+e_{(\phi)}^{t}(r)}{n^{(\phi)}\left(r, \phi ; \theta_{\mathrm{o}}\right) e_{(t)}^{\phi}(r)+e_{(\phi)}^{\phi}(r)}$,

where $n^{(a)}=p^{(a)} / p^{(t)}$ is a unit three-vector in the direction of photon emission. We choose the local frame attached to an observer with the tetrad $e_{(a)}^{\alpha}$ (here, we assumed that $p_{\mathrm{t}}=-1$ and $\left.p_{\phi}=l\right)$.

The simplest assumption one can impose is that photons are emitted "straight" toward the observer,

$n^{(\phi)}\left(r, \phi ; \theta_{\mathrm{o}}\right)=-\sin \phi \sin \theta_{\mathrm{o}}$.

This was adopted by Zhang \& Bao (1991) and in many subsequent papers, neglecting the effect of light bending. This turns out to provide a sufficiently accurate description in many astrophysically relatistic situations; however, Zhang \& Bao further assume that the photons move straight in the direction to an observer in the local frame co-moving with the disc (i.e. the tetrad $e_{(\mathrm{a})}^{\mu}$ in Eq. (10) corresponds to a Keplerian observer and vector $n^{(a)}$ is expressed in this tetrad). In this way they also neglect the aberration due to rotation of the disc, which enlarges the error especially at small inclination (pole-on view). The resulting formula for the redshift is

$g_{\mathrm{ZB}}\left(r, \phi ; \theta_{\mathrm{O}}\right)=\left[\frac{r-2}{r(r-3)}\right]^{1 / 2}\left[(r-2)^{1 / 2}-\sin \phi \sin \theta_{\mathrm{O}}\right]$.
Extremal values occur on a ring $r=$ const. $\phi \equiv \phi_{ \pm}=\mp 90^{\circ}$,

$g_{\mathrm{ZB}}^{ \pm}\left(r, \theta_{\mathrm{o}}\right)=\left[\frac{r-2}{r(r-3)}\right]^{1 / 2}\left[(r-2)^{1 / 2} \pm \sin \theta_{\mathrm{o}}\right]$.

These approximate formulae suffer from excessive inaccuracy in some regions of the parameter space. We computed these fractional errors and illustrate them in three plots. In Fig. 1 we plot the maximum and minimum relative errors of the redshift factor $\delta_{ \pm} g\left(r, \theta_{0}\right)$ and the relative error of the extremal values of $g$-factor $\delta g_{ \pm}\left(r, \theta_{0}\right)$ on a ring $r=r_{\mathrm{ms}}$ (marginally stable orbit). Figure 2 shows the dependence on radius for $\theta_{0}=60^{\circ}$. Figure 3 shows the azimuthal dependence of $g$-factor itself and its relative error.

The main source of error is not the neglect of light bending and, indeed, considerably better results are achieved if we perform this calculation in the local frame of a static observer, still neglecting the light bending. In this approximation the tetrad of a static observer is used in Eq. (10) and the redshift function is

$g_{\mathrm{sl}}\left(r, \phi ; \theta_{\mathrm{o}}\right)=\left[\frac{(r-2)(r-3)}{r}\right]^{1 / 2} \frac{1}{(r-2)^{1 / 2}+\sin \phi \sin \theta_{\mathrm{o}}}$,

and its extremal values on a ring, which again occur at $\phi_{ \pm}=\mp 90^{\circ}$, are

$g_{\mathrm{sl}}^{ \pm}\left(r, \theta_{\mathrm{o}}\right)=\left[\frac{(r-2)(r-3)}{r}\right]^{1 / 2} \frac{1}{(r-2)^{1 / 2} \mp \sin \theta_{\mathrm{o}}}$.

This approach is very successful at estimating the extremal values (see the left panel in bottom row of Fig. 1) but the relative errors of the redshift factor itself remain non-negligible.

Finally, the approach can be refined by assuming that photon trajectories are curved by gravity and adopting a suitable approximation to their shapes (Beloborodov's 2002). Then the azimuthal component of the directional three-vector $n^{(a)}$ is (see Appendix A)

$n^{(\phi)}\left(r, \phi ; \theta_{0}\right)=-\left[\frac{1-\cos ^{2} \alpha}{1-\cos ^{2} \psi}\right]^{1 / 2} \sin \phi \sin \theta_{0}$

with $\cos \alpha=1-(1-\cos \psi)(1-2 / r)$ and $\cos \psi=\cos \phi \sin \theta_{0}$. The angle $\alpha$ is the angle that local static observer measures between the radial direction and the direction of emission. The redshift factor is

$$
\begin{aligned}
& g_{\mathrm{B}}\left(r, \phi ; \theta_{\mathrm{O}}\right)= \\
& \frac{[r(r-3)]^{1 / 2}}{r+\left[r-2+4\left(1+\cos \phi \sin \theta_{\mathrm{o}}\right)^{-1}\right]^{1 / 2} \sin \phi \sin \theta_{\mathrm{O}}} .
\end{aligned}
$$

Expressions for the extremal values in this case are summarized in the previous section, see Eqs. (2)-(7).

\section{Comparison with observations}

Chandra and XMM-Newton have discovered narrow and sometimes transient features in the 5 to $7 \mathrm{keV}$ energy range. Dovčiak et al. (2004a) argued that these lines may be the blue horn of an iron line profile from an emitting annulus in the accretion disc, a situation which could arise if an X-ray flare just 

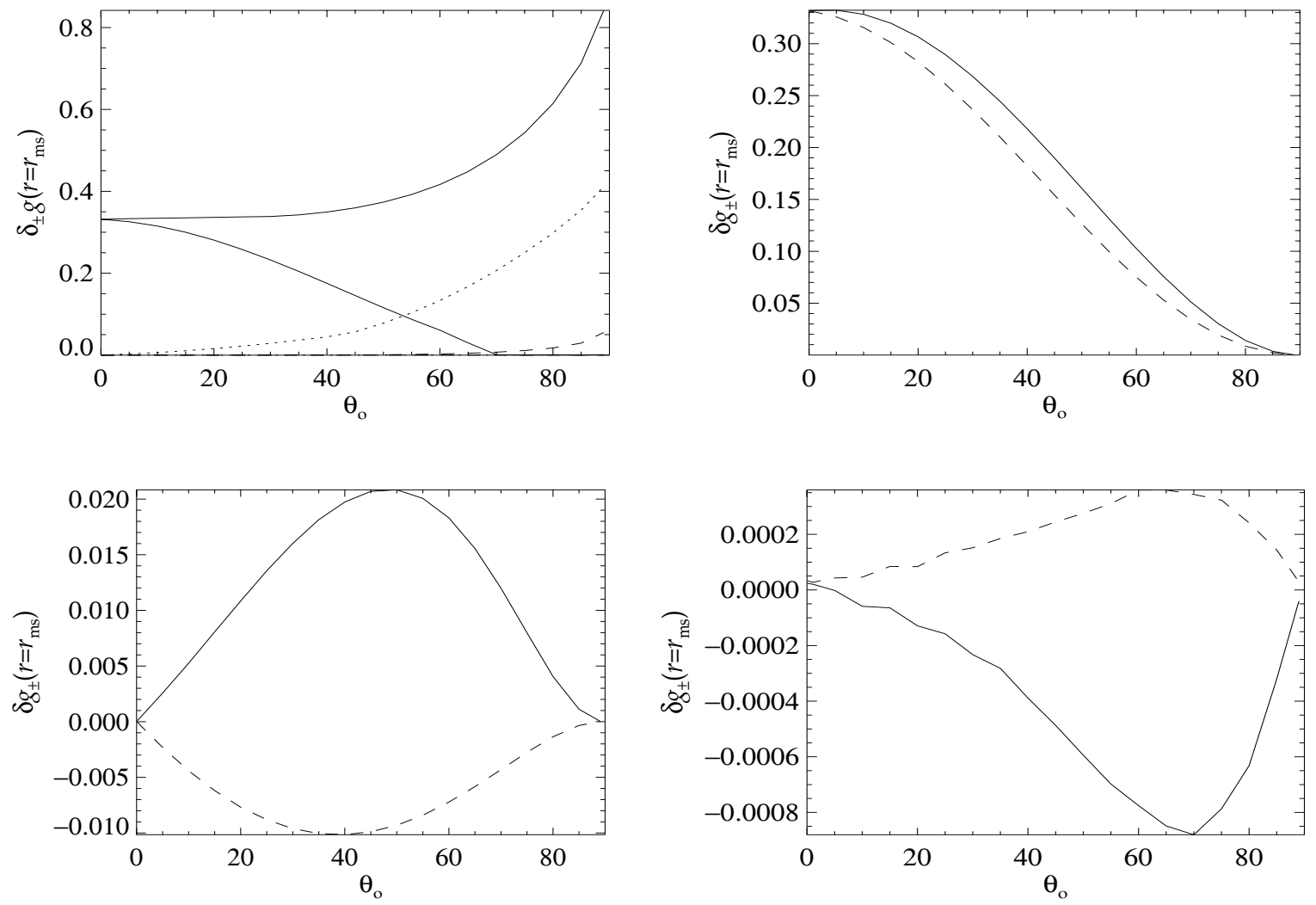

Fig. 1. Accuracy assessment for different approximation formulae. (i) Top row - left panel: the dependence of the maximum and minimum relative error $\delta_{ \pm} g\left(r, \theta_{\mathrm{o}}\right)$ of the $g$-factor on observer's inclination angle $\theta_{\mathrm{o}}$ for a narrow ring, $r=r_{\mathrm{ms}}$. Solid lines refer to Eq. (12) (the upper one is for maximum, the lower one is for minimum); the dotted line refers to Eq. (14); the dashed line is for Eq. (17). The approximation formula (12) is the worst of the three cases in the sense that it has a significant non-zero value of the minimum error. This is due to neglecting aberration. The relative error $\delta g=\left|g_{\text {approx }}-g\right| / g$ is determined with respect to exact (numerically computed) values. (ii) Top row - right panel: the relative error $\delta g_{ \pm}\left(r, \theta_{\mathrm{o}}\right)$ of the extremal values of $g$-factor for a ring $r=r_{\mathrm{ms}}$ as a function of observer's inclination angle. The relative error $\delta g_{ \pm}=\left(g_{\text {approx. }}^{ \pm}-g_{ \pm}\right) / g_{ \pm}$of the extremal values is again determined with respect to exact values from equation of geodesic. The solid/dashed lines are for maximum/minimum of $g$-factor; both lines are for the approximation (13). (iii) Bottom row - left panel: the same as top right but with the approximation (15). (iv) Bottom row - right panel: the same as top right but for the approximation (2).
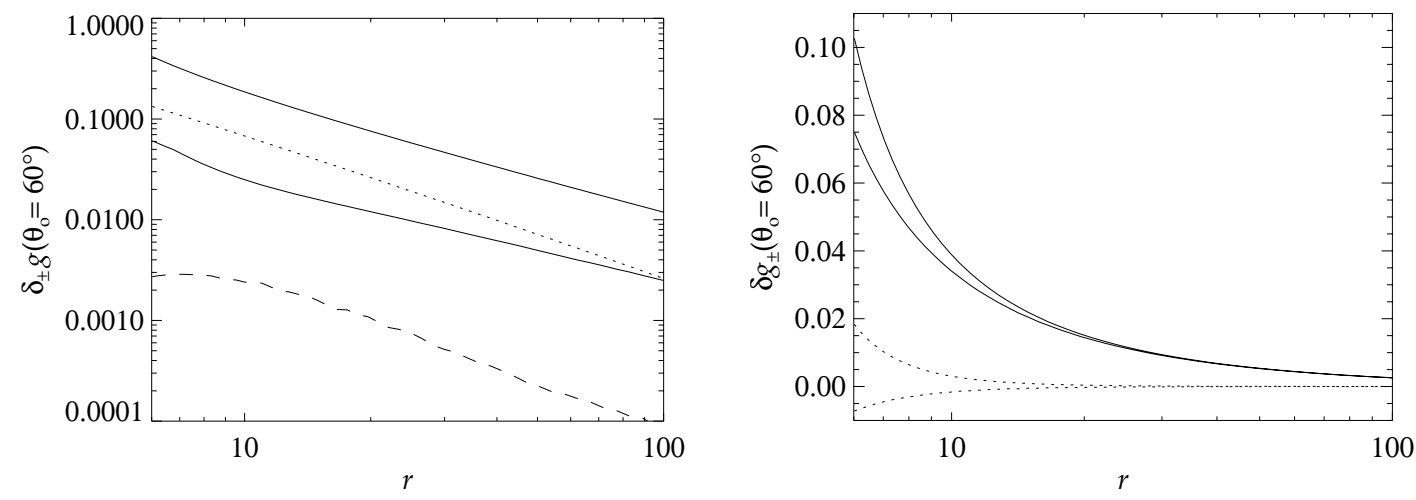

Fig. 2. Left: the dependence of the maximum and minimum relative error $\delta_{ \pm} g\left(r, \theta_{\mathrm{o}}\right)$ of $g$-factor on the radius for inclination angle $\theta_{\mathrm{o}}=60^{\circ}$, in logarithmic scale. Solid lines are for the approximation (12) (upper for the maximum, lower for the minimum), dotted line is for the approximation (14) and dashed line is for the approximation (17). Right: the relative error $\delta g_{ \pm}\left(r, \theta_{\mathrm{o}}\right)$ of the extremal values of $g$-factor. The solid lines are for Eq. (13) and the dashed lines are for Eq. (15). Upper lines show the relative error of $g_{+}$, lower lines are for the relative error of $g_{-}$. The relative error for the approximation (2) is better than $0.1 \%$ and thus it is not plotted here.

above the disc survives a non-negligible fraction of the orbital period or more.

These features offer the possibility to measure the black hole mass (see discussion in Dovčiak et al. 2004a), and indeed Iwasawa et al. (2004) applied this method to the
Seyfert 1 galaxy, NGC 3516. The quality of data, however, is in most cases insufficient for this purpose. For the time being, and waiting for the next generation of X-ray satellites like Constellation-X and XEUS, we estimate the orbiting spot parameters. 

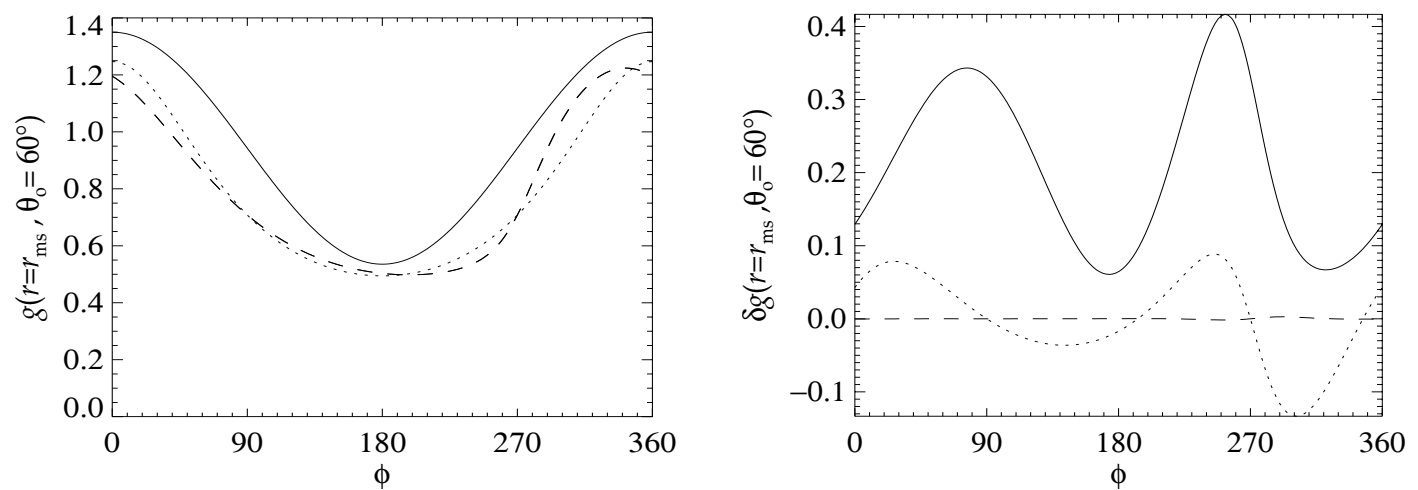

Fig. 3. Left: $g$-factor as a function of the azimuthal coordinate $\phi$ for $r=r_{\mathrm{ms}}, \theta_{\mathrm{o}}=60^{\circ}$. The solid line is for Eq. (12), the dotted line is for Eq. (14) and the dashed line is for Eq. (17). The exact numerical solution is also plotted by the dashed line and cannot be distinguished from the approximation (17) in this figure. Right: as on the left but for the relative error of $\delta g$ for the three cases of approximation formulae. Notice that the error of the best one, Eq. (17), is virtually zero.
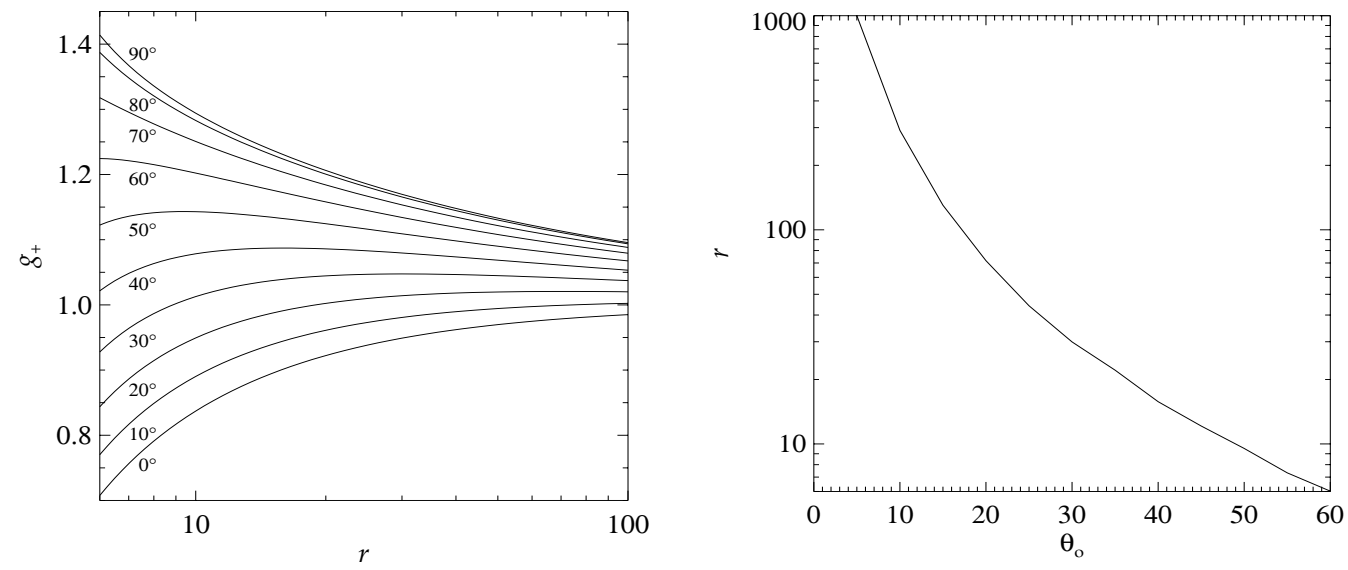

Fig. 4. Left: the maximum redshift factor $g_{+}(r)$ as a function of the radius. Different curves are parametrized by inclination angle $\theta_{0}$. Right: the radius at which the function $g_{+}(r)$ is maximum as a function of $\theta_{0}$. For $\theta_{0} \gtrsim 60^{\circ}$ the maximum occurs at marginally stable orbit where the inner edge of the disc is assumed.

In Fig. 4 (left panel) we show the maximum energy shift $g_{+}(r)$, which corresponds to the blue edge of the line and the peak flux when lensing is neglected or unimportant. Only direct image photons were considered (higher-order images can be important only for equatorial inclinations of $\theta_{\mathrm{o}} \sim 90^{\circ}$; see below). In this case the energy shift is given by Eq. (2). We checked that the exact value (based on the full geodesic equation and the ray tracing method) for the energy shift is indistinguishable from the approximation adopted in this figure. This plot permits determination of ranges of allowed $\left(r, \theta_{0}\right)$ pairs from the measured energy of the narrow feature. A complementary plot is given in the right panel where we show radius at which the maximum energy shift occurs for a given value of inclination angle $\theta=\theta_{\mathrm{o}}$.

One can ask whether the lensing effect, which we have neglected in our present discussion, could change the conclusion about the observed energy of the Doppler boosted horn of a relativistic line. The reason is that for higher inclination angles $\left(\theta_{0} \gtrsim 60^{\circ}\right)$ the lensing effect and bending of light rays (that changes the projected area of the spot) can play an important role: thus the dominating feature need not be a blue horn associated with the maximum shift of energy but, instead, a new peak of the maximum observed flux that should arise redwards of $g_{+}(r)$ (see e.g. Matt et al. 1993). We have therefore employed a ray-tracing code (Dovčiak et al. 2004b) taking all these general-relativity effects into account, and examined time-dependent model spectra. Indeed a new peak is formed by photons arriving from another azimuth (different from $-90^{\circ}$ ) of the spot orbit. The secondary maximum is in the point where the effect of energy shift, lensing, projection area and time delay combine to produce the maximum amplification. However, the time delay, and especially the function $g(r, \phi)$, vary rapidly across the region of the disc most affected by lensing. As a result, the new peak becomes spread over a broad range of energy and does not form a narrow line. In other words, even if the lensed feature may have a large equivalent width and can contribute significantly to the total photometric flux, this effect is not relevant for our present discussion of the narrow line origin, as the resulting feature is not narrow at all.

In Table 1 we summarize the values of $r$ and $\theta_{\mathrm{o}}$ for the most convincing observational cases known. For all features a solution in which the orbiting spot is in the innermost stable orbit for a Schwarzschild black hole, i.e $r=6$, is acceptable. The inclination angle is generally expected to be low or moderate (the inner accretion disc is presumably aligned with the outer torus). If the features we discuss originate at or near the 
Table 1. The narrow features detected so far in AGN which can be interpreted as the blue horns of a $6.4 \mathrm{keV}$ iron line arising from an orbiting spot.

\begin{tabular}{c|ccccl}
\hline \hline Source & $E_{\text {line }}[\mathrm{keV}]$ & $r\left[G M / c^{2}\right]$ & $\theta_{\mathrm{o}}[\mathrm{deg}]$ & $r_{\theta_{0}=\text { const. }}\left[G M / c^{2}\right]$ & Reference \\
\hline NGC 3516 & 5.57 & $6-12$ & $0-23$ & $6.6\left(\theta_{\mathrm{o}}=20^{\circ}\right)$ & Turner et al. (2002) \\
& 6.22 & $6-50$ & $0-35$ & $12.4\left(\theta_{\mathrm{o}}=20^{\circ}\right)$ & \\
& 6.53 & $>6$ & $20-40$ & $56 / 93\left(\theta_{\mathrm{o}}=20^{\circ}\right)$ & \\
\hline ESO 198-G024 & 5.70 & $6-14$ & $0-26$ & $7.2\left(\theta_{\mathrm{o}}=20^{\circ}\right)$ & Guainazzi (2003) \\
& 5.96 & $6-21$ & $0-30$ & $8.9\left(\theta_{\mathrm{o}}=20^{\circ}\right)$ & Dovčiak et al. (2004a) \\
\hline NGC 7314 & 5.84 & $6-17$ & $0-28$ & $6.3\left(\theta_{0}=27^{\circ}\right)$ & Yaqoob et al. (2003) \\
& 6.61 & $>6$ & $27-41$ & $20 / 93\left(\theta_{0}=27^{\circ}\right)$ & \\
\hline Mrk 766 & 5.60 & $6-12.5$ & $0-24$ & $6.7\left(\theta_{\mathrm{o}}=20^{\circ}\right)$ & Turner et al. (2004) \\
& 5.75 & $6-15$ & $0-27$ & $7.4\left(\theta_{\mathrm{o}}=20^{\circ}\right)$ & \\
\hline ESO 113-G010 & 5.40 & $6-10$ & $0-20$ & $6\left(\theta_{\mathrm{o}}=20^{\circ}\right)$ & Porquet et al. (2004) \\
\hline
\end{tabular}

innermost stable orbit, the corresponding inclination angle is the upper value in the table. Naturally, as this angle must be the same for different features of the same source, the actual allowed range is the intersection of the various ranges: $20-23^{\circ}$ (NGC 3516), 0-26 (ESO 198-G024), 27-28 (NGC 7314), $0-24^{\circ}$ (Mrk 766). Assuming, therefore, the same inclination angle for a given source, and assuming this angle to be $20^{\circ}$ for all sources but one, the corresponding radii are listed in the fourth column of the table. For NGC 7314, if the two features are both the blue horn of an annular emission, then the inclination angle is constrained to be between $27^{\circ}$ and $28^{\circ}$; for this source an angle of $27^{\circ}$ has been assumed. For the only two features that are blueward of the line rest energy, the resulting radii could not be determined uniquely. This is explained by the fact that the line of $g=$ const. can intersect the curve $g_{+}(r)$ at two different radii (see Fig. 4).

The values of the parameters in the table have been derived assuming a rest-frame energy of the line $E_{0} \sim 6.4 \mathrm{keV}$, as appropriate for neutral matter. However, the iron may be ionized and the corresponding $E_{0}$ higher. The maximum possible energy is for H-like iron, and is $E_{0} \sim 6.96 \mathrm{keV}$ (the line is a doublet again). Because in this case the redshift has to be larger, the upper limits to the radius and the inclination angle are reduced. For instance, for the reddest feature (that of ESO 113-G010) the allowed ranges are now $6 \lesssim r \lesssim 7.6$ for the dimensionless radius and $\theta_{\mathrm{o}} \lesssim 11^{\circ}$ for the inclination.

As discussed above, for high inclination angles the maximum flux could occur not in correspondence with the blue peak, as we assumed in this paper, but instead with the moment of the spot crossing a caustic at an intermediate phase. In this case the resulting inclination comes out larger. We have explored and rejected this lensing effect as the origin of narrow lines not only due to the large inclination, but also because the width of the resulting spectral feature exceeds substantially the width of the blue peak. In no case do the observed features require a spinning black hole, as a solution with $r>6$ is always found.

\section{Discussion}

Variability properties contain a wealth of information on accreting black holes, but much of it is lost in the process of time integration in the detector. Several ways have been proposed to measure the parameters of accreting black holes, which rely on the source variability: for example, limits on the black-hole mass and its rate of rotation can be derived from time delays between variations of the emission-line strength and of the continuum, and from temporal changes of the observed emission lines. Time-dependent spectra have not yet provided the anticipated breakthrough in our understanding; they still remain a challenge for theorists and observers alike. This is caused by difficulties and non-uniquenesses in the interpretation of the available dynamical spectra. On the side of observation a tradeoff must be accepted between energy and temporal resolution which also compromises the usefulness of timing spectroscopy. That is why we search for sharp features, and the narrow redshifted lines are promising.

Here we showed that a simple and accurate formula can be given for the estimate of the energy shift. The analytical formula circumvents the necessity of numerical integration of photon paths and can be straightforwardly used to determine the orbiting spot parameters from the measured energy of the narrow feature, with an accuracy that is more than sufficient. The use of this formula implies the assumptions that the feature corresponds to the blue peak in profile, that the effect of blackhole rotation is negligible and that the disc is essentially planar.

Acknowledgements. The authors of this paper have been supported by the Czech Science Foundation under grants 205/03/H144 (T.P.), 205/05/P525 (M.D.) and 205/03/0902 (V.K.), and by Italian Ministry of Research under grant COFIN-03-02-23 (G.M.). Financial support from the Academy of Sciences of the Czech Republic is also gratefully acknowledged (grant IAA 300030510). The Astronomical Institute has been operated under the project AV0Z10030501.

\section{Appendix A: Direction of photon emission}

In the Schwarzschild metric a ray can be defined by three points: the origin of the coordinate system (which coincides with the center of the black hole), location of the spot (in the disc plane), and the observer (at radial infinity). See Fig. A.1 for the geometrical setup. Then the directional vector $n^{(a)}$ of a photon can be expressed with respect to the local static frame. 

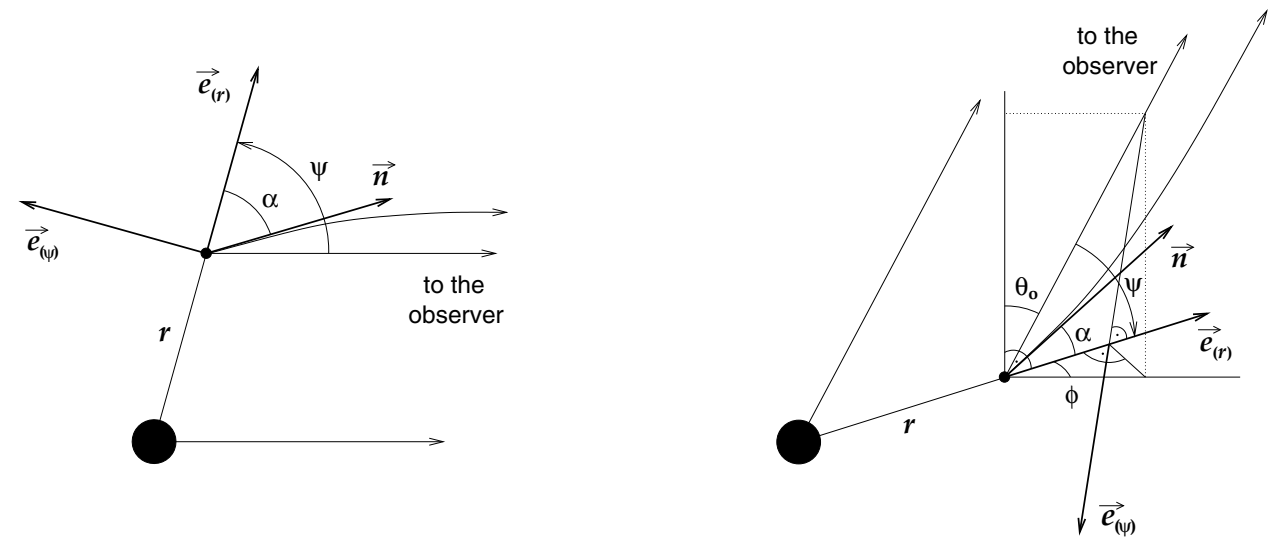

Fig. A.1. A schematic representation of the plane of photon motion (left), and the definition of angles and directions in Schwarzschild coordinates $(r, \theta, \phi)(r i g h t)$. The direction vector $\boldsymbol{n}$ is tangent to the photon ray projected into three-space.

We have employed Beloborodov's (2002) approximation of light rays. One can write

$n^{\mu}=\cos \alpha e_{(r)}^{\mu}-\left(1-\cos ^{2} \alpha\right)^{1 / 2} e_{(\psi)}^{\mu}$,

where the unit vector $e_{(\psi)}^{\mu}$ is perpendicular to the tetrad vector $e_{(r)}^{\mu}$ and lies in the plane of the photon's motion. Thus (see the right panel in Fig. A.1)

$e_{(\psi)}^{\mu}=r^{-1}\left(1-\cos ^{2} \psi\right)^{-1 / 2}\left(0,0, \cos \theta_{0}, \sin \phi \sin \theta_{0}\right)$

with $\cos \psi=\cos \phi \sin \theta_{0}$. The components of the directional vector $n^{(a)}=n^{\mu} e_{(a) \mu}$ in a local static frame are

$n^{(r)}=\cos \alpha$,

$n^{(\theta)}=-\left[\frac{1-\cos ^{2} \alpha}{1-\cos ^{2} \psi}\right]^{1 / 2} \cos \theta_{0}$,

$n^{(\phi)}=-\left[\frac{1-\cos ^{2} \alpha}{1-\cos ^{2} \psi}\right]^{1 / 2} \sin \phi \sin \theta_{\mathrm{o}}$.

Here, we have used the tetrad of a static observer located in the equatorial plane:

$e_{(t)}^{\mu}=(1-2 / r)^{-1 / 2}(1,0,0,0)$,

$e_{(r)}^{\mu}=(1-2 / r)^{1 / 2}(0,1,0,0)$,

$e_{(\theta)}^{\mu}=r^{-1}(0,0,1,0)$,

$e_{(\phi)}^{\mu}=r^{-1}(0,0,0,1)$.

\section{References}

Bao, G. 1992, A\&A, 257, 594

Bao, G., Hadrava, P., \& Østgaard, E. 1994, ApJ, 435, 55

Bao, G., \& Stuchlík, Z. 1993, ApJ, 400, 163

Beckwith, K., \& Done, C. 2004, MNRAS, 352, 353

Beckwith, K., \& Done, C. 2005, MNRAS, 359, 1217

Beloborodov, A. M. 2002, ApJ, 586, L85

Bianchi, S., Matt, G., Balestra, I., Guainazzi, M., \& Perola, G. C. 2004, A\&A, 422, 65

Bromley, B. C., Chen, K., \& Miller, W. A. 1997, ApJ, 475, 57

Čadež, A., Brajnik, M., Gomboc, A., Calvani, M., \& Fanton, C. 2003, A\&A, 403, 29

Chandrasekhar, S. 1992, The Mathematical Theory of Black Holes (New York: Oxford Univ. Press)
Cunningham, C. T., \& Bardeen, J. M. 1973, ApJ, 183, 273

Czerny, B., Różańska, A., Dovčiak, M., Karas, V., \& Dumont, A.-M. 2004, A\&A, 420, 1

Della Ceca, R., Ballo, L., Braito, V., \& Maccacaro, T. 2005, ApJ, 627, 706

Dovčiak, M., Bianchi, S., Guainazzi, M., Karas, V., \& Matt, G. 2004a, MNRAS, 350, 745

Dovčiak, M., Karas, V., \& Yaqoob, T. 2004b, ApJS, 153, 205

Fabian, A. C., Iwasawa, K., Reynolds, C. S., \& Young, A. J. 2000, PASP, 112,1145

Fabian, A. C., Rees, M. J., Stella, L., \& White, N. E. 1989, MNRAS, 238, 729

Gallo, L. C., Boller, T., Brandt, W. N., Fabian, A. C., \& Vaughan, S. 2004, MNRAS, 355, 330

Gerbal, D., \& Pelat, D. 1981, A\&A, 95, 18

Guainazzi, M. 2003, A\&A, 401, 903

Haardt, F., Maraschi, L., \& Ghisellini, G. 1994, ApJ, 432, 95

Hartnoll, S. A., \& Blackman, E. G. 2002, MNRAS, 332, L1

Iwasawa, K., Miniutti, G., \& Fabian, A. C. 2004, MNRAS, 355, 1073

Karas, V., Lanza, A., \& Vokrouhlický, D. 1995, ApJ, 440, 108

Karas, V., Martocchia, A., \& Subr, L. 2001, PASJ, 53, 189

Kojima, Y. 1991, MNRAS, 250, 629

Laor, A. 1991, ApJ, 376, 90

Martocchia, A., Karas, V., \& Matt, G. 2000, MNRAS, 312, 817

Martocchia, A., Matt, G., Karas, V., Belloni, T., \& Feroci, M. 2002, A\&A, 387, 215

Matt, G., Perola, G. C., \& Stella, L. 1993, A\&A, 267, 643

McKernan, B., \& Yaqoob, T. 2004, ApJ, 608, 157

Misner, C. W., Thorne, K. S., \& Wheeler, J. A. 1973, Gravitation (San Francisco: Freeman)

Porquet, D., Reeves, J. N., Uttley, P., \& Turner, T. J. 2004, A\&A, 427, 101

Poutanen, J., \& Fabian, A. 1999, MNRAS, 306, L31

Reynolds, C. S., \& Nowak, M. A. 2003, PhR, 377, 389

Tanaka, Y., et al. 1995, Nature, 375, 659

Thompson, A. C., Attwood, D. T., Gullikson, E. M., et al. 2001, X-Ray Data Booklet (Berkeley: Lawrence Berkeley National Laboratory)

Turner, T. J., Mushotzky, R. F., Yaqoob, T., et al. 2002, ApJ, 574, L123

Turner, T. J., Kraemer, S. B., \& Reeves, J. N. 2004, ApJ, 603, 62

Turner, T. J., Kraemer, S. B., George, I. M., Reeves, J. N., \& Bottorff, M. C. 2005 , ApJ, 618, 155

Usui, F., Nishida, S., \& Eriguchi, Y. 1998, MNRAS, 301, 721

Viergutz, S. U. 1993, A\&A, 272, 355

Yaqoob, T., George, I. M., Kallman, T. R., et al. 2003, ApJ, 596, 85

Zakharov, A. F., \& Repin, S. V. 2003, A\&A, 406, 7

Zhang, X.-H., \& Bao, G. 1991, A\&A, 246, 21 